\title{
Development of a New Acquisition Scheme for Energy-Filtering Transmission Electron Microscopy Spectrum-Imaging toward Quantification
}

\author{
M. Watanabe* and F.I. Allen** \\ * Dept of Materials Science and Engineering, Lehigh University, Bethlehem. PA 18015. \\ ** National Center for Electron Microscopy, Lawrence Berkeley National Laboratory, Berkeley, \\ CA94720.
}

Energy-filtering transmission electron microscopy (EFTEM) enables the determination of spatially resolved elemental distributions over a large field of view with a relatively high spatial resolution, e.g. $\sim 1 \mathrm{~nm}$ at $200 \mathrm{kV}$ [e.g., ref 1]. This elemental mapping technique can be expanded by implementing EFTEM spectrum-imaging (SI), in which a series of filtered images is acquired at different energy-loss positions offering improved background (BG) subtraction, optimized core-loss signal integration and advanced spectral processing [e.g. refs 2, 3]. However, in the EFTEM-SI approach the signal intensities in each filtered image are lower than those obtained by the conventional three-window method since individual filtered images in EFTEM SI are usually acquired with a narrower energy slit and for a shorter acquisition time. Such weak signals in filtered images are easily influenced by CCD conditions such as dark current (DC) and shot noise, and degrade following spatial-drift correction and BG subtraction.

Recently, Heil and Kohl proposed an improved drift-correction scheme for EFTEM elemental mapping which uses a zero-loss (ZL) image-series acquired simultaneously with the core-loss (CL) image-series [4]. In the work presented here, a new acquisition scheme for EFTEM SI, called SmartEFTEM-SI, has been developed expanding on the aforementioned procedure by incorporating a number of improvements. The improved features in the SmartEFTEM-SI approach are (1) initialization of the CCD DC prior to acquisition in order to refresh the CCD, (2) measurement of the CCD DC prior to every filtered-image acquisition to compensate for DC modulation, (3) acquisition of the CL image-series from the higher energy-loss side to prevent CCD saturation, (4) multiple-frame acquisition of individual filtered images to improve the signal-to-noise ratio, (5) acquisition of a ZL image between successive filtered images for superior spatial drift-correction, and (6) acquisition of a low-loss (LL) image-series after the CL image-series acquisition for further advanced spectral-processing and quantification. The acquisition procedure of SmartEFTEM-SI with the full set of options (1)-(6) is summarized in Fig. 1. When applying the SmartEFTEM-SI method using the full option set, four data cubes (image stacks) are stored: ZL, CL, LL and DC image-series. During acquisition all images are stored into a file stream for efficient memory use. By applying several post-acquisition processing techniques including spatial drift-correction via the ZL image-series, CL and LL SI-datasets can be obtained. The SmartEFTEM-SI approach works well both in omega-filter instruments (JEOL JEM-2200FS and Zeiss Libra 200) and in any instrument with a Gatan imaging filter (GIF) operating under the DigitalMicrograph platform.

An example of the SmartEFTEM-SI method applied to a boron nitride (BN) specimen is shown in Fig. 2. A boron map (c) was obtain ed from a region of varying thickness (bright-field ZL image shown in (a), relative thickness map shown in (b)) by SmartEFTEM-SI and power-law BG subtraction. Then, using a LL SI-dataset, Fourier-log deconvolution was applied to the BG-subtracted CL SI. Fig. 2(d) compares spectra in the vicinity of the boron K-edge extracted 
from CL SI-datasets before and after deconvolution, showing that the effect of plural scattering can be removed from the EFTEM-SI dataset. Furthermore, a boron density map (e) was obtained by dividing the deconvoluted signal-map by the LL signal. Finally, a map of the number of boron atoms (f) was quantified by dividing the density map by the partial cross-section of the boron K-edge. The SmartEFTEM-SI method thus offers absolute quantification of elemental maps.

\section{References}

[1] R.F. Egerton, Electron Energy-loss Spectroscopy in the Electron Microscope, Plenumn, NY, (1996).

[2] J. Mayer, et al., Micron, 28 (1997), 361.

[3] P.J. Thomas and P.A. Midgley, Ultramicrosc., 88 (2001), 179.

[4] T. Heil and H. Kohl, Ultramicrosc., 110 (2010), 748.

[5] The author (MW) wish es to acknowledge financial support from the NSF through grant DMR-0804528.

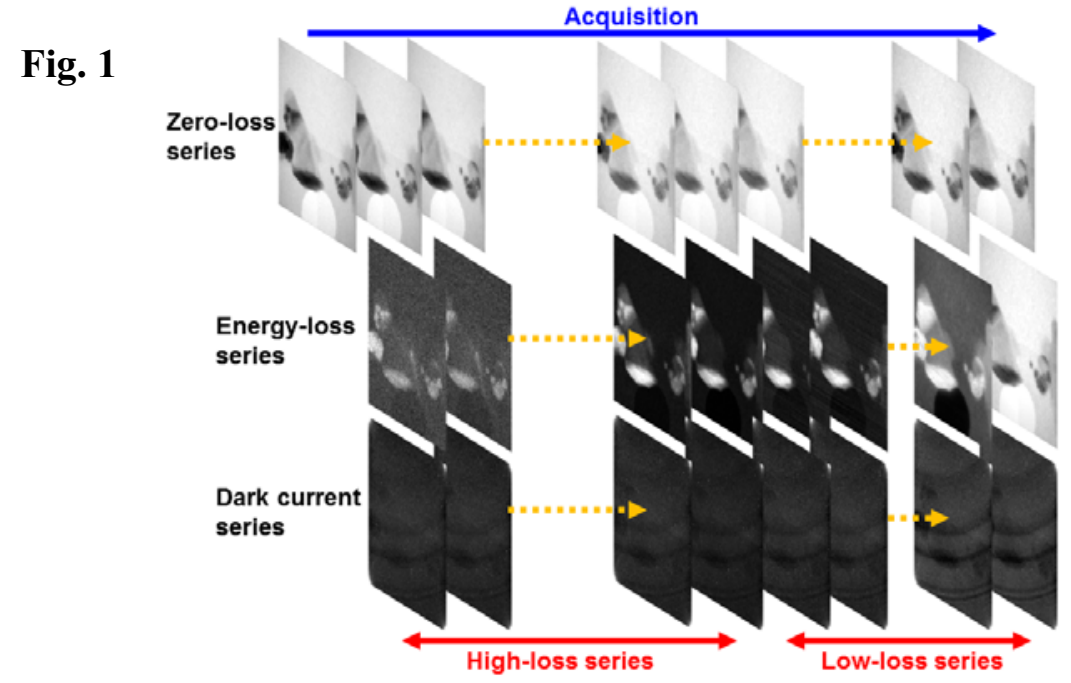

Fig. 2
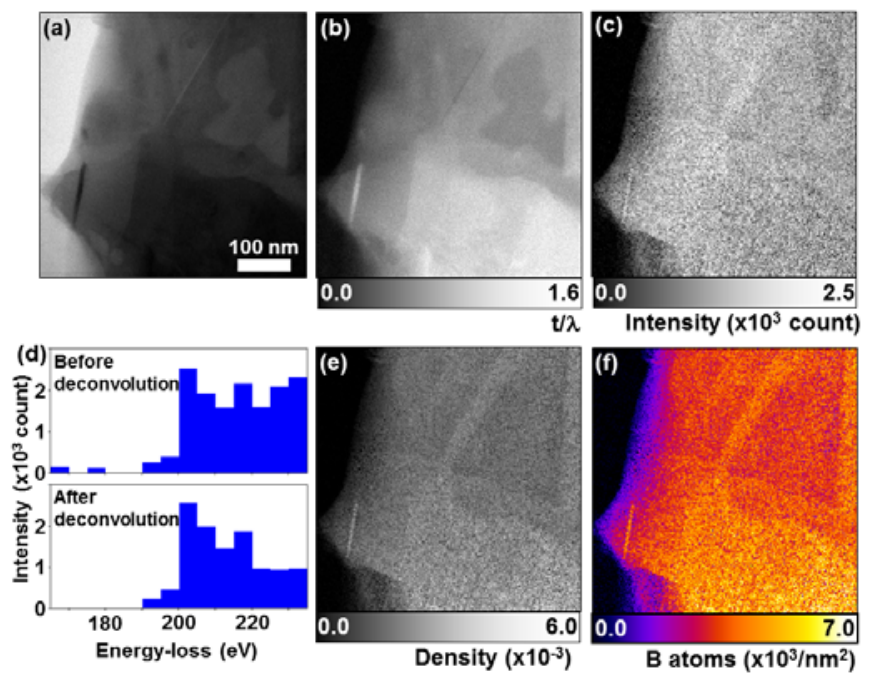

Figure 1: Summary of acquisition procedure of the SmartEFTEM-SI method.

Figure 2 Application of SmartEFTEM-SI to BN: (a) BF-ZL, (b) relative thickness, (c) B map,

(d) effect of Fourier-log deconvolution, (e) B density and (f) a number of B atoms in $\mathrm{nm}^{2}$. 